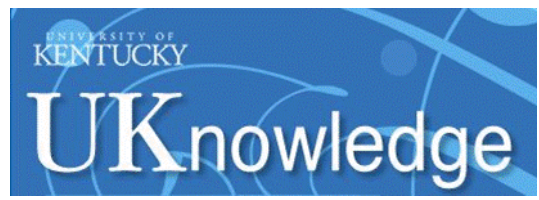

University of Kentucky

UKnowledge

\title{
Systematically Exploring the Effects of Pole Count on the Performance and Cost Limits of UltraHigh Efficiency Fractional hp Axial Flux PM Machines
}

\author{
Narges Taran \\ University of Kentucky, narges.taran@uky.edu \\ Vandana Rallabandi \\ University of Kentucky, vandana.rallabandi@uky.edu \\ Greg Heins \\ Regal Beloit Corporation, Australia \\ Dan M. Ionel \\ University of Kentucky, dan.ionel@uky.edu
}

Follow this and additional works at: https://uknowledge.uky.edu/peik_facpub

Part of the Power and Energy Commons

Right click to open a feedback form in a new tab to let us know how this document benefits you.

\section{Repository Citation}

Taran, Narges; Rallabandi, Vandana; Heins, Greg; and Ionel, Dan M., "Systematically Exploring the Effects of Pole Count on the Performance and Cost Limits of UltraHigh Efficiency Fractional hp Axial Flux PM Machines" (2020). Power and Energy Institute of Kentucky Faculty Publications. 7.

https://uknowledge.uky.edu/peik_facpub/7

This Article is brought to you for free and open access by the Power and Energy Institute of Kentucky at UKnowledge. It has been accepted for inclusion in Power and Energy Institute of Kentucky Faculty Publications by an authorized administrator of UKnowledge. For more information, please contact UKnowledge@lsv.uky.edu. 


\title{
Systematically Exploring the Effects of Pole Count on the Performance and Cost Limits of UltraHigh Efficiency Fractional hp Axial Flux PM Machines
}

\author{
Digital Object Identifier (DOI) \\ https://doi.org/10.1109/TIA.2019.2943814
}

\section{Notes/Citation Information}

Published in IEEE Transactions on Industry Applications, v. 56 , issue: 1.

(C) 2019 IEEE Copyright Notice. "Personal use of this material is permitted. Permission from IEEE must be obtained for all other uses, in any current or future media, including reprinting/republishing this material for advertising or promotional purposes, creating new collective works, for resale or redistribution to servers or lists, or reuse of any copyrighted component of this work in other works."

The document available for download is the authors' manuscript version that is accepted for publication. The final published version is copyrighted by IEEE and will be available as: N. Taran, V. Rallabandi, G. Heins, and D. M. lonel, "Systematically Exploring the Effects of Pole Count on the Performance and the Cost Limits of Ultra-high Efficiency Fractional hp Axial Flux PM Machines," in IEEE Transactionson Industry Applications, 2019, 10 p. 


\title{
Systematically Exploring the Effects of Pole Count on the Performance and Cost Limits of Ultra-high Efficiency Fractional hp Axial Flux PM Machines
}

\author{
Narges Taran, Student Member, IEEE, Vandana Rallabandi, Senior Member, IEEE, \\ Greg Heins, Member, IEEE, Dan M. Ionel, Fellow, IEEE
}

\begin{abstract}
Optimizing the design of electric machines is a vital step in ensuring the economical use of active materials. The three-dimensional flux paths in axial flux PM (AFPM) machines necessitate the use of computationally expensive 3D electromagnetic analysis. Furthermore, a large number of design evaluations is required to find the optimum, causing the total computation time to be excessively long. In view of this, a two-level surrogate assisted algorithm capable of handling such expensive optimization problems is introduced, which substantially reduces the number of FEA evaluations to less than 200 while conventional algorithms require thousands of designs to be analyzed. The proposed algorithm is employed to optimally design an AFPM machine within a specified envelope, and to identify the limits of cost and efficiency. In order to obtain these limits, the variables' ranges are assigned to be as wide as possible, resulting in a vast design space, the study of which was enabled by the developed special algorithm. Additionally, optimized designs with different rotor polarities are systematically compared in order to form the basis for a set of generalized design rules.
\end{abstract}

Index Terms-Axial flux permanent magnet, surrogate assisted, multi-objective, optimization, number of poles, 3D FEA, SPM.

\section{INTRODUCTION}

The design and optimization of axial flux permanent magnet (AFPM) machines are of great interest due to many of their advantages such as higher torque density and efficiency. Their disc shape makes it practical to achieve designs with a larger diameter and a higher number of poles. The possibility to have a higher pole count leads to potential performance improvements. Determining the number of rotor poles and subsequently stator slots greatly impacts the outcomes of the electric machine design optimization [1]. This paper is a systematic investigation of those impacts for a surface mounted AFPM machine.

Recently, the fractional slot concentrated winding (FSCW) has attracted attention for its application in surface mounted PM machines [2]-[4]. Its main advantages include shorter end coils, a simple structure, and improved fault tolerance. The use of double layer FSCW, as opposed to its single layer counterpart, can mitigate the core and PM losses. Also, in

N. Taran and D. M. Ionel are with the SPARK Laboratory, the Department of Electrical and Computer Engineering, University of Kentucky, Lexington, KY 40506 USA (e-mails: narges.taran@uky.edu; dan.ionel@uky. edu). V. Rallabandi was with the SPARK Laboratory, ECE Department, University of Kentucky, and is now with GE Research, Niskayuna, NY (e-mail: vandana.rallabandi@ieee.org). G. Heins is with the Regal Beloit Corporation, Australia, Rowville, VIC 3178, Australia (e-mail: greg.heins@ regalbeloit.com).

Authors' manuscript accepted for publication. The final published version is copyrighted by IEEE and will be available as: N. Taran, V. Rallabandi, G. Heins, and D. M. Ionel, "Systematically Exploring the Effects of Pole Count on the Performance and the Cost Limits of Ultra-high Efficiency Fractional hp Axial Flux PM Machines," IEEE Transactions on Industry Applications, 2019, 10p. O2019 IEEE Copyright Notice. "Personal use of this material is permitted. Permission from IEEE must be obtained for all other uses, in any current or future media, including reprinting/republishing this material for advertising or promotional purposes, creating new collective works, for resale or redistribution to servers or lists, or reuse of any copyrighted component of this work in other works." case of axial flux configurations, the double layer winding can yield an increased active diameter within a constrained total diameter, due to the reduction in coil thickness.

Studies on surface mounted PM machines (SPM) with a concentrated winding provide the principles for selecting the best combinations for the number of slots and poles. They propose a slot per pole per phase less than or equal to 0.5 [5], $N_{s}=N_{p} \pm 1$ or $N_{s}=N_{p} \pm 2$ [6]-[9], and $N_{s}>N_{p}$ [10], where $N_{s}$ and $N_{r}$ are the number of slots and poles, respectively. Based on the above guidelines, four slot/pole combinations $(12 / 10,24 / 20,36 / 30,48 / 40)$ with a double layer concentrated winding are selected.

The main shortcomings of the studies conducted on the effect of slot/pole combinations is that they rely on simplistic assumptions, such as a constant yoke thickness, and the use of analytical approaches and/or 2D models for machines with 3D flux paths [3], [11]-[15], which results in comparing topologies that are not truly optimal designs for their pole count.

This paper is an expanded follow-up to a previous conference paper by the same authors [16]. Together the current and the previous conference paper provide substantial contributions to the subject matter by: systematically investigating the optimal design and pole count selection of a surface mounted AFPM machine; employing an algorithm capable of solving optimization problems with 3D FEA design evaluations and a very wide search space; and proposing a method for interpreting the Pareto front and selecting the optimal design in a multi-objective problem.

The optimization method is a two-level surrogate assisted algorithm that employs a combination of differential evolution (DE) algorithm, kriging meta models as surrogate interpolations, and 3D FEA. Through this approach, thousands of designs are evaluated with a relatively accurate surrogate model, while the most promising designs are analyzed using the very high fidelity 3D finite element method.

The paper is structured as follows: the next section introduces the AFPM machine specifications and its 3D parametric model. The selected objectives and geometrical variables are explained. Section III introduces the optimization algorithm. The optimization results are discussed in section IV, which also includes a proposed systematic approach for design selection and interpretation of the Pareto front of a multi-objective optimization. The results are validated experimentally in sections $\mathrm{V}$. The study concludes in section VI. 


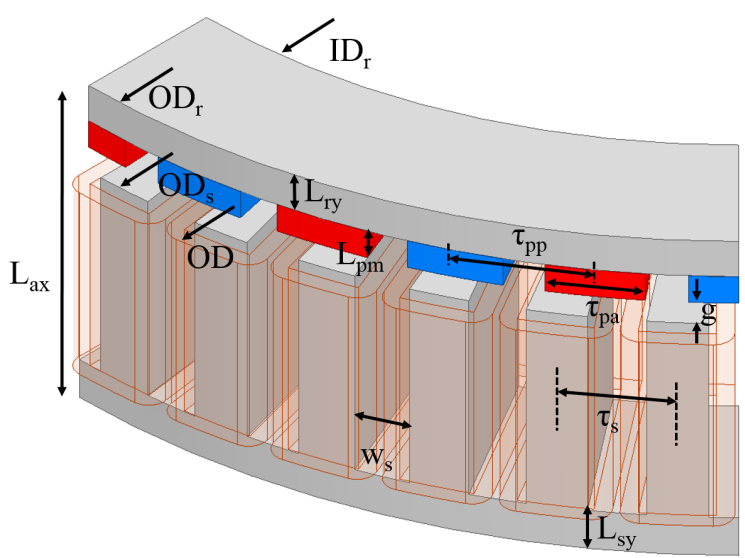

Fig. 1. The geometrical independent variables included in the process of design optimization. The variables are introduced to the algorithm as ratios.

TABLE I

INDEPENDENT OPTIMIZATION VARIABLES AND CORRESPONDING LIMITS, EXEMPLIFIED FOR THE CONFIGURATION WITH 40 POLES.

\begin{tabular}{llcc}
\hline Variable & Description & Min & Max \\
\hline$g$ & Air-gap $[\mathrm{mm}]$ & 1.50 & 4.00 \\
$k_{r y}$ & rotor yoke ratio $=\frac{L_{r y}}{L_{a x}}$ & 0.07 & 0.25 \\
$k_{s y}$ & stator yoke ratio $=\frac{L_{s y}}{L_{a x}}$ & 0.07 & 0.25 \\
$k_{p m}$ & magnet length ratio $=\frac{L_{p m}}{L_{a x}}$ & 0.06 & 0.25 \\
$k_{d s}$ & split ratio $=\frac{I D_{s}}{O D_{s}}\left(O D_{r}-O D_{s}\right)$ & 0.48 & 0.80 \\
$k_{o h}$ & over hang ratio $=\frac{\left(O D-O D_{s}\right)}{\left(O D w_{s}\right.}$ & -1.00 & 1.00 \\
$k_{s w}$ & slot width to slot pitch ratio $=\frac{w_{s, i d}}{\tau_{s}}$ & 0.45 & 0.90 \\
$k_{p}$ & pole arc to pole pitch ratio $=\frac{\tau_{p a}}{\tau_{p p}}$ & 0.60 & 0.95 \\
\hline
\end{tabular}

\section{Problem Formulation For AnAlysis AND OPTIMIZATION}

The axial flux machine under study is designed to produce the rated torque of $5.4 \mathrm{Nm}$ at $1050 \mathrm{rpm}$. The optimization study is comprehensive and considers all possible designs within the limitations of this problem, i.e. geometrical dimensions, mechanical limits, etc., in order to reach the absolute limit of each objective function. As designs within the widest applicable range of variables are included, a reference design to improve upon is not considered in this study.

The envelope size, including axial length and total outer diameter are constant and fixed at their maximum value while number of poles and other geometrical variables are investigated to achieve the most favorable design. The optimal design problem is elaborated in the following.

\section{A. Objectives}

Considering that cost competitiveness and design efficiency are the most important criteria from the perspective of manufacturers and customers, the optimization objectives are considered to be the total loss and cost [17]. The two optimization objective functions are defined for the total loss, $F_{l}$, and active material cost, $F_{c}$ :

$$
\begin{aligned}
& F_{l}=W_{C u}+W_{c}+W_{p m}, \\
& F_{c}=m_{c}+3 \cdot m_{C u}+24 \cdot m_{p m},
\end{aligned}
$$

where $W_{C u}, W_{c}$, and $W_{p m}$ stand for the copper, stator core, and PM eddy losses. The preliminary studies showed that the rotor core loss is a negligible portion of the total loss in this study, and is therefore not considered in the analysis. The low rotor core losses can be attributed to the relatively low fundamental frequency, as well as reduced rotor core flux density harmonics due to application of surface mounted PMs. The magnet losses can be reduced by segmentation.

The total mass of the stator and rotor core are represented with $m_{c}$, and the copper and magnet mass, with $m_{C u}$ and $m_{p m}$, respectively. The mass is calculated in $\mathrm{kg}$ and the steel cost per $\mathrm{kg}$ is considered as the one-unit reference [18]. This per-unitized method of defining the cost function makes the calculations independent of the impact of using various vendors and currencies, while still including a correct representation of the cost trends needed for design comparison.

It is expected that the best compromise design varies for different pole counts. One of the goals of this study is to investigate those varying factors in order to identify generalized rules for a suitable initial design of SPM machines with different polarities.

Generally, as the number of poles and slots increases, the copper loss decreases, due to the reduced end winding length. On the other hand, the increase in the fundamental frequency causes more core losses. Therefore, a pole count which achieves a proper trade-off between copper and core losses, for a given application and dimensional constraints exists, and should be identified. The methodology developed in this paper can also be utilized to identify the number of poles most suitable for a specified application.

\section{B. Parametric 3D FEA Models}

The AFPM machines to be optimized are single stator single rotor configurations with surface mounted Neodymium magnets and concentrated non-overlapping windings. The stator core is laminated. In order to include the effect of varying number of poles, different optimization studies are conducted for four slot/pole combinations i.e. 12/10, 24/20, $36 / 30$, and 48/40. The total axial height and the total outer diameter including the end coils are kept the same in all the studied topologies. Figure 1 illustrates the 3D parametric model, exemplified for the 40 pole configuration. All of the FEA results are obtained from time-transient 3D models with motion.

\section{Optimization Variables}

The search space for the optimal design is assigned to be very large, i.e., the ranges of the optimization variables are as wide as possible, with only geometrical limitations taken into account, to include the absolute limits of the minimum cost and loss achievable. Therefore, the variables are limited either to prevent the intersection of various geometrical components or to address mechanical constraints such as the minimum airgap or yoke thickness.

Eight geometrical variables are selected as optimization variables, as illustrated in Fig. 1 and Table I. The flux lines distribution for the 40 pole topology, as an instance, 

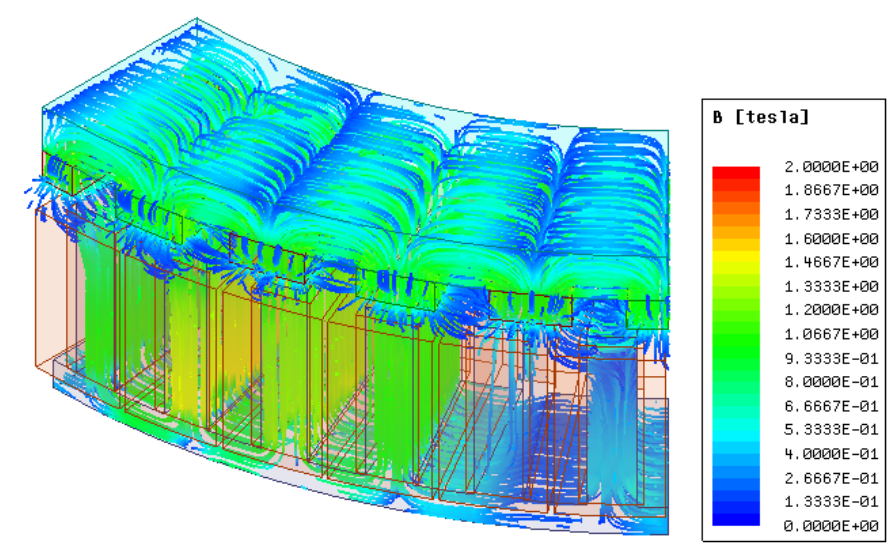

Fig. 2. The 3D flux lines distribution of an exemplified design with 40 poles.

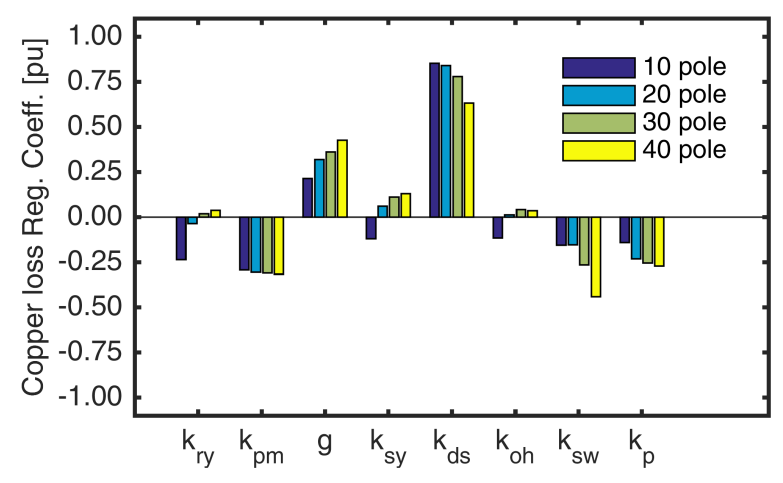

Fig. 3. The effects of optimization variables on dc copper loss of designs with different pole count, represented by per-unitized regression coefficients.

is presented in Fig. 2. The variables are defined as ratios to ensure their independence. Only the air-gap is defined as an absolute value, in $\mathrm{mm}$. The variables in the axial direction are divided by the total axial length, $L_{a x}$. The slot depth is calculated for a constant overall axial length.

The over-hang ratio, $k_{o h}$, is defined to take values between -1 and +1 . The positive values indicate over-hang, that is, the magnet radial length is greater than the stator radial length with the same average radius. The negative values represent underhang, that is, the magnet radial length is less than the stator radial length with the same average radius. Taking over-hang into account, particularly in cost sensitive designs, is helpful in potential PM volume reduction. It is ensured that all designs produce the rated torque at the rated speed through a twopass analysis. First the design is analyzed using a preassigned current density. Then, based on the produced torque and the required torque, the current density is adjusted and the design is re-evaluated to ensure the rated torque is obtained.

\section{Sensitivity Studies}

The effects of the optimization variables on the performance of the designs with different number of poles are investigated by studying regression coefficients, which are obtained by conducting a design of experiments study. Negative and positive regression coefficients indicate that the absolute value of the response reduces and increases, respectively, upon increase

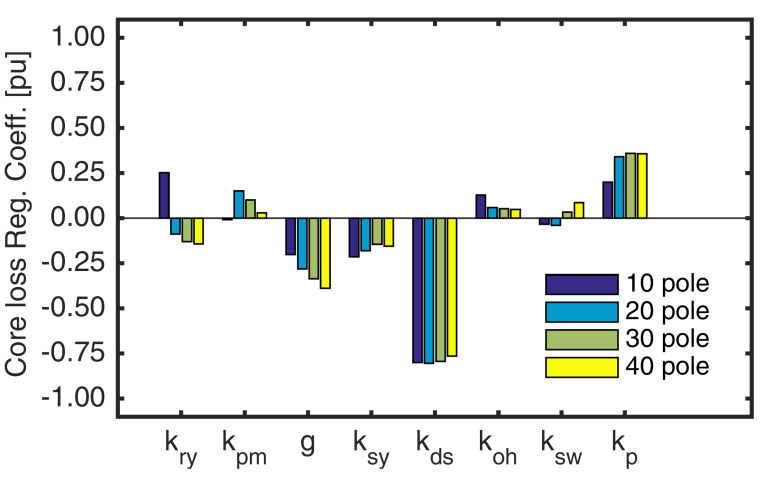

Fig. 4. The effects of optimization variables on core loss of designs with different pole count, represented by per-unitized regression coefficients.

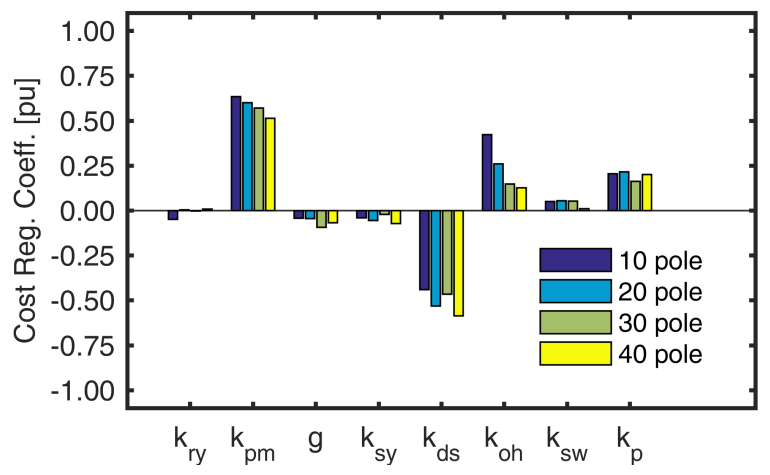

Fig. 5. The effects of optimization variables on cost function of designs with different pole count, represented by per-unitized regression coefficients.

in the corresponding factor. The larger the magnitude of the regression coefficients, the more influential the factor.

The influence of variations in the optimization variables on dc copper loss, stator core loss, and cost function are illustrated in (Fig. 3, Fig. 4, and Fig. 5). The magnet loss comprises a smaller fraction of the total losses. In the case of the specific ratings and envelope constraints in this study, it may be observed from these figures that the general trend is that the copper losses increase upon increasing the air-gap $(g)$, and split ratio $\left(k_{d s}\right)$. On the other hand, by increasing magnet thickness $\left(k_{p m}\right)$, slot width $\left(k_{s w}\right)$ and pole arc $\left(k_{p}\right)$, the copper loss reduces. This is attributed to the higher magnetic loading achieved by an increase in these parameters, and therefore, a lower current density can be employed to produce the rated torque. The rotor yoke $\left(k_{r y}\right)$ appears to be influential only in case of the machine with the lowest pole count, which may be owing to its higher saturation, considering the larger pole pitch.

It is observed that the degree of the impacts varies for different pole counts. With fixed axial length, the increase in yoke thickness can be achieved by reducing the slot depth. This may alter the leakage pattern and/or the harmonic spectrum and hence core losses in a non-straightforward way. Figure 5 shows that the effect of overhang ratio $\left(k_{o h}\right)$ is more considerable in cost function and more so in case of the machines with lower number of poles. This design of experiments based 


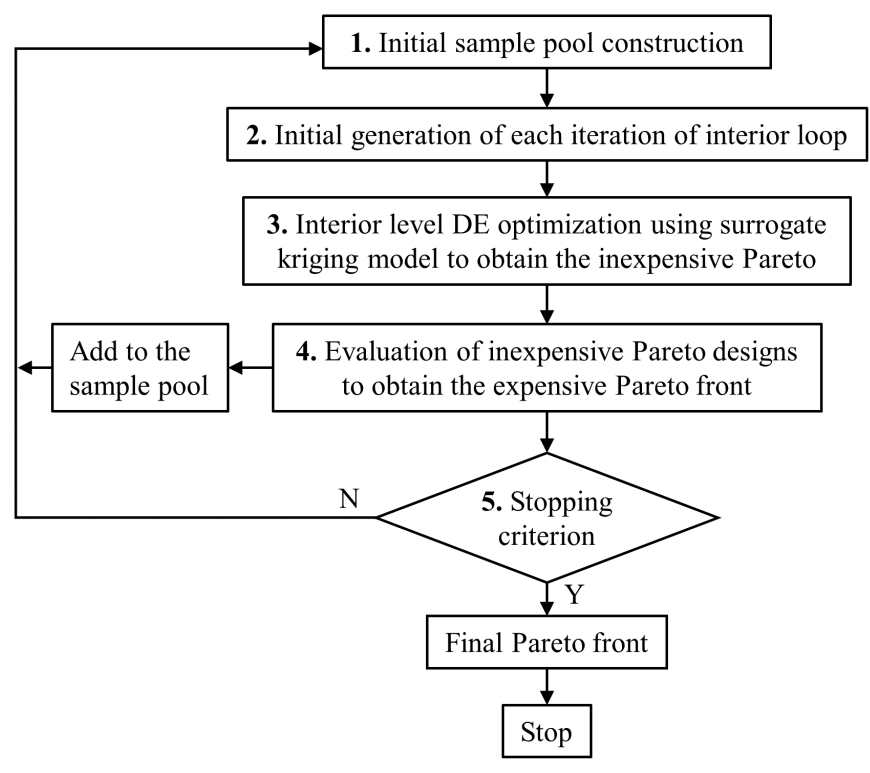

(a)

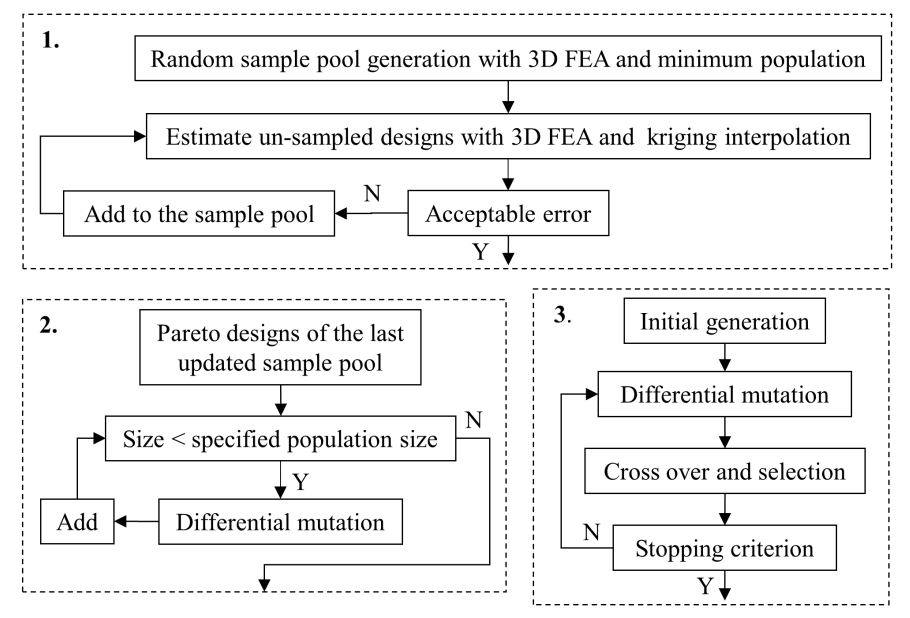

(b)

Fig. 6. (a) The flowchart for the two-level surrogate assisted algorithm, proposed for multi-objective optimization of electric machines with 3D FEA models. (b) The details of blocks 1, 2, and 3.

study shows that the selected studied variables have significant effects on objectives. Therefore, the optimization is performed with all eight discussed variables.

\section{Two-LEVEl Surrogate Assisted Optimization}

The magnetic flux for an AFPM machine is in both axial and circumferential directions. The flux leakage occurs in three dimensions, (Fig. 2). Also, the tooth width variation in the radial direction makes the stator core in the inner diameter prone to saturation. Two-dimensional or quasi-3D models cannot capture the effects of end coils and overhang. These are some of the reasons necessitating the use of computationally expensive 3D models for AFPM machines.

Generally, thousands of design evaluations are required to identify the Pareto front [17]. Moreover, the full exploration of a wide design space, as is undertaken in this paper, would typically require even more designs to be evaluated. For these
TABLE II

NUMBER OF GENERATIONS, FE MODELS, AND KRIGING SURROGATE MODELS FOR EACH OPTIMIZATION STUDY.

\begin{tabular}{lrrrr}
\hline Poles & 40 & 30 & 20 & 10 \\
\hline Generations & 5 & 7 & 5 & 9 \\
FE models & 211 & 207 & 164 & 300 \\
Kriging models & 3130 & 3120 & 2040 & 5640 \\
\hline
\end{tabular}

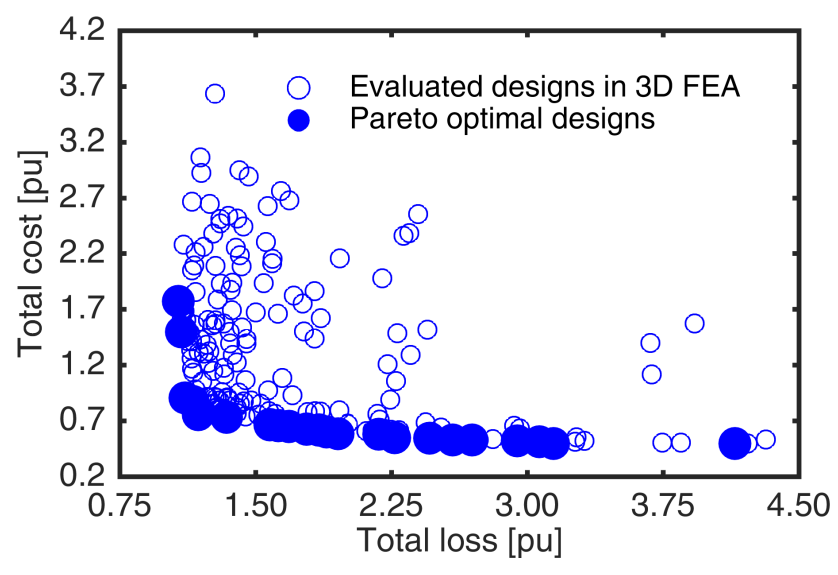

Fig. 7. All designs evaluated in 3D FEA for the 30 pole configuration and the Pareto optimal designs represented by filled circles.

reasons, the use of 3D FEA models with conventional algorithms is simply unaffordable. Surrogate assisted optimization algorithms make it feasible to evaluate a larger number of designs. In this paper, a new two-level surrogate assisted differential evolution multi-objective optimization (SAMODE) is employed in order to identify the Pareto front accurately with a minimum number of expensive design evaluations. This algorithm reduces the required number of FEA design evaluations from thousands to less than two hundred.

The flowchart representing the new optimization algorithm is illustrated in Fig. 6. This is a two-level surrogate-assisted algorithm taking advantage of differential evolution and kriging models. The flowchart is composed of an exterior and an interior level loop. The exterior loop is an evolutionary algorithm, replacing the regeneration step with an interior loop. The interior loop is based on a multi-objective differential evolution (MODE) algorithm that employs surrogate models for function evaluations [19]. A summary of the optimization process is provided in the following.

The first step of the optimization algorithm is to construct the initial sample pool. This may be done with random designs or design of experiments (DoE). For optimization problems with larger number of variables and a wide design space, such as the study at hand, DoE requires a large number of designs for inclusion in the sample pool in order to take possible nonlinearities into account. Therefore, in this study, random designs are used to construct the initial sample pool. The resolution of the sample pool is subsequently increased only in the promising areas of the search space.

After generating the minimum sample size, the performance of a limited number of unsampled designs, say ten designs, 
is evaluated with the surrogate model and also simulated with FEA and an estimation error is calculated. If the error is larger than a preset limit, e.g. 5\%, these designs are added to the pool and a new batch of unsampled designs are evaluated. This process constantly increases the sample pool size until satisfactory estimations are achieved.

The next step is to select the initial generation. For each iteration of interior loop, the initial population is set to be the Pareto front designs of the latest sample pool. Then, the interior level optimization is performed using, in this case, conventional DE methods with inexpensive surrogate models as its function evaluation tool. The output of this is an estimated Pareto front. It is expected to have some estimation errors for the Pareto front designs performance, to correct which, the Pareto front designs are evaluated with 3D FEA and replaced their approximate estimations suggested by the surrogate models. The interior loop optimization is performed again, this time with the updated sample pool. This continues until the termination criteria are reached.

Multi-objective optimizations usually set a maximum number of function evaluations or maximum number of generations as the termination criterion. For the algorithms that converge to the optima very fast, this criterion can cause many dispensable generations which is vital to avoid. In this study, in order to reduce expensive evaluations, a negligible improvement in three representative points of the Pareto front, for a few consecutive generations, will terminate the optimization.

The surrogate model used in this algorithm is Gaussian process prediction [20], known as kriging in geostatics. The kriging surrogate model is a local fitting model that, unlike conventional curve fitting methods, does not fit a global polynomial function. The kriging model puts more weight on sampled data points in the vicinity of the un-sampled data, providing nonlinear estimations that are more accurate even for the outliers. Kriging surrogate models can be divided into trend and residual components [21],

$$
\begin{aligned}
& \hat{Y}=\hat{X} \beta+r^{T} R^{-1}(Y-X \beta) ; \\
& r_{i}=\exp \left[-\Sigma_{t=1}^{k} \theta_{t}\left|\hat{x_{t}}-x_{i, t}\right|^{2}\right], \\
& R_{i, j}=\exp \left[-\Sigma_{t=1}^{k} \theta_{t}\left|x_{i, t}-x_{j, t}\right|^{2}\right] ; i, j=1, \ldots, n,
\end{aligned}
$$

where $\hat{Y}$ is the response to be predicted based on the known sampled data points, i.e. $X$ and $Y . \beta$ is the matrix of regression coefficients that can be obtained using methods such as least squares. $n$ is the number of samples and $k$ the number of variables. Kriging weights, $r^{T}$ and $R^{-1}$ are derived from the covariance function or semivariogram and maximum likelihood estimation (MLE).

The two level layout provides an approach to evaluate only the most promising designs with expensive 3D FEAs in the exterior loop, while the interior loop provides an approach for evaluating thousands of designs using inexpensive surrogate interpolations. This algorithm, unlike existing surrogate assisted algorithms, does not solely rely on estimated values, and has a dynamic sample pool that increases the initial sample pool size until the estimation error is sufficiently small. Such an implementation avoids unnecessary expensive design eval-
TABLE III

THE PER-UNIT VALUE OF REPRESENTATIVE OPTIMUM DESIGNS FOR

\begin{tabular}{|c|c|c|c|c|c|}
\hline \multicolumn{2}{|c|}{ Number of poles } & 40 & 30 & 20 & 10 \\
\hline \multirow{4}{*}{ 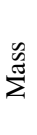 } & Copper & 0.15 & 0.18 & 0.22 & 0.22 \\
\hline & Steel & 0.58 & 0.60 & 0.74 & 0.94 \\
\hline & PM & 0.03 & 0.03 & 0.04 & 0.05 \\
\hline & Total & 0.76 & 0.81 & 1.00 & 1.21 \\
\hline \multirow{4}{*}{$\begin{array}{l}\vec{n} \\
\dot{0}\end{array}$} & Copper & 0.20 & 0.24 & 0.29 & 0.29 \\
\hline & Steel & 0.26 & 0.26 & 0.33 & 0.41 \\
\hline & PM & 0.29 & 0.26 & 0.38 & 0.50 \\
\hline & Total & 0.75 & 0.77 & 1.00 & 1.21 \\
\hline \multirow{4}{*}{$\begin{array}{l}\tilde{\alpha} \\
0 \\
0\end{array}$} & Copper & 0.71 & 0.67 & 0.49 & 0.75 \\
\hline & Core & 0.63 & 0.43 & 0.37 & 0.22 \\
\hline & PM & 0.03 & 0.07 & 0.14 & 0.17 \\
\hline & Total & 1.36 & 1.17 & 1.00 & 1.14 \\
\hline \multirow{4}{*}{\multicolumn{2}{|c|}{$\begin{array}{l}\text { Emag. efficiency }[\%] \\
\text { Power density }[\mathrm{W} / \mathrm{kg}] \\
\text { Torque density }[\mathrm{Nm} / \mathrm{kg}]\end{array}$}} & 92.7 & 93.7 & 94.6 & 93.9 \\
\hline & & 217.5 & 205.5 & 165.4 & 136.8 \\
\hline & & 2.0 & 1.9 & 1.5 & 1.2 \\
\hline & & 0.79 & 0.86 & 0.93 & 0.87 \\
\hline
\end{tabular}
DIFFERENT POLE COUNTS. THE TOTAL MASS, COST, AND LOSS OF THE SELECTED 20 POLE DESIGN REPRESENT THE BASE.

uations while the kriging model resolution gradually improves for designs closer to the Pareto front.

\section{OPtimizATION RESUltS AND Discussion}

The two-level surrogate assisted optimization is performed on the AFPM machines with different number of poles. For all cases, the population size is set at ten designs per generation. The base optimization method is DE, shown in Fig. 6b, block number 3 , which requires scaling factor and cross over probability; both are assigned to be 0.5 .

The number of generations is dynamic and not fixed, as the optimization terminates once further improvement is not achieved. The number of total FEA runs is also variable because it depends on factors such as the acceptable sample pool resolution, the number of generations, and the number of new proposed promising designs in each generation. The number of generations, FE models, and krigign surrogate model design evaluations for each optimization set-up is provided in Table II.

As expected, because each generation includes hundreds of design candidates evaluated with ultra-fast kriging meta models, even the total number of generations is much lower than for typical DE optimization algorithms, resulting in a substantial reduction of the computational effort. Only a relatively low number of 3D FE models are employed, each solved with the ANSYS/Maxwell software in approx. 30 minutes on a desktop PC workstation with 10 cores, Intel(R) Xeon(R) CPU E5-2687W v3 @ 3.10 GHz, and much faster on HPC systems with large scale parallel processing capabilities. As an example for the 30 pole machine, Fig. 7 includes all the 207 candidates evaluated with 3D FEA and for clarity does not include the results for the 3120 Kriging models.

\section{A. Optimal Design Selection}

The final product of such a multi-objective optimization, after reaching termination criteria, is a set of non-dominated 
designs, referred to as Pareto optimal designs. For minimization problems, a design is Pareto optimal if there exists no other feasible design within the defined search space which would decrease one objective without causing a concurrent increase in at least one other objective [22]. Mathematically, designs variables' vector $\overrightarrow{x^{*}}$ is called a Pareto optimal design, if no other vector $\mathrm{x}$ exists that satisfies both the following conditions [23]:

$$
\begin{aligned}
& f_{j}(\vec{x}) \leq f_{j}\left(\overrightarrow{x^{*}}\right) \quad \text { for all } j \in(1,2, \ldots, m) ; \\
& f_{j}(\vec{x})<f_{j}\left(\overrightarrow{x^{*}}\right) \quad \text { for at least one } j \in(1,2, \ldots, m) .
\end{aligned}
$$

where $f$ represents the objective function and $m$ is the number of objective functions, for this study $m=2$. An example Pareto front obtained for the 30 pole topology and 2 objectives of this study is plotted in Fig. 7.

The final design should be selected among the Pareto optimal designs. The Pareto designs closer to the knee region of the Pareto front provide a trade-off between the two objectives while the designs further away from the knee point represent the superiority of one of the objectives over the other. The designs that are located more to the left side represent those high in efficiency and cost, while the designs on the right feature low cost and high loss

The relation between the two objectives can be captured by fitting a curve on the Pareto front. The general format of the fitted curve can be described as

$$
\hat{f}_{2}=\frac{a}{\hat{f}_{1}-b}+c
$$

where $\hat{f}_{1}$ and $\hat{f}_{2}$ are the two objective functions on the fitted curve (loss and cost, respectively, in this study), and constants $a, b$, and $c$ are positive values that can be assigned based on the exact shape of the obtained Pareto front.

The final design selection can be performed by quantifying the superiority of objectives over one another. This quantification can be expressed by a factor $T$; any percentage-wise further improvement in one objective should cause less than $T$ times deterioration in the other, that is:

$$
\frac{\Delta \hat{f_{t 1}}}{\hat{f_{t 1}}}=-\frac{\Delta \hat{f_{t 2}}}{\hat{f_{t 2}}} \times T \stackrel{\Delta f \rightarrow 0}{\Longrightarrow} \frac{d \hat{f_{t 2}}}{d \hat{f_{t 1}}}=-\frac{1}{T} \times \frac{\hat{f_{t 2}}}{\hat{f_{t 1}}} .
$$

Equation (5) in combination with (4) can be solved for $\hat{f_{t 1}}$ and $\hat{f_{t 2}}$ that are the the objective values of the desired design. The closest design on Pareto to $\left(\hat{f_{t 1}}, \hat{f_{t 2}}\right)$ can be selected as the final design.

The knee point can be defined as the point where $T=1$. This means any percentage-wise improvement in one of the objectives results into the exact amount of percentage-wise deterioration in the other, and is therefore representative of a best-compromise design. The calculated knee point is marked with a star in Fig. 8. Two other examples, prioritizing loss and cost, are also included and marked. In one example, with $T=0.2$, further percentage-wise reduction of loss will cause at least 5 times $(1 / 0.2=5)$ more percentage-wise increase in cost. As another example, where $T=5$, further reduction in cost leads to at least 5 times more increase in the loss.

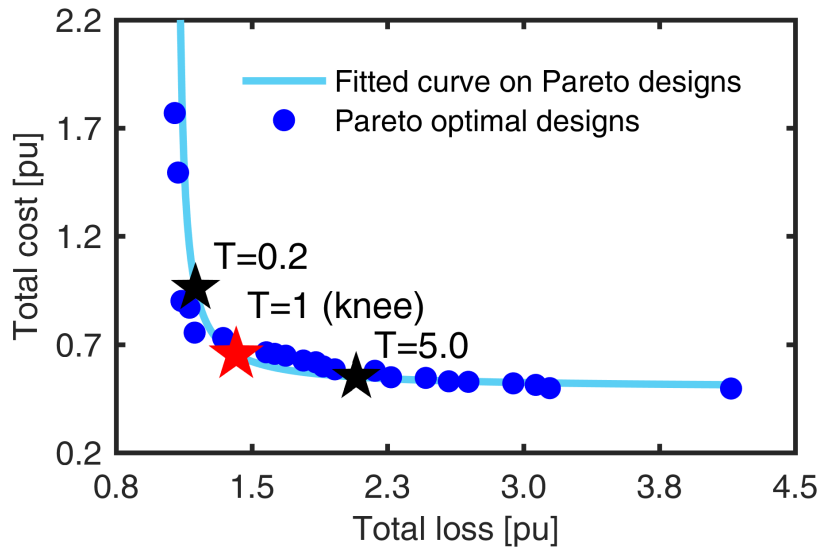

Fig. 8. The fitted curve on the Pareto front and the calculated knee point, based on (5), marked with the red star symbol $(\star)$. The point $T=0.2$ is where further reduction in loss causes at least $5\left(\frac{1}{0.2}=5\right)$ times more increase in the cost. The point $T=5$ is where further reduction of cost causes at least 5 times more increase in loss.

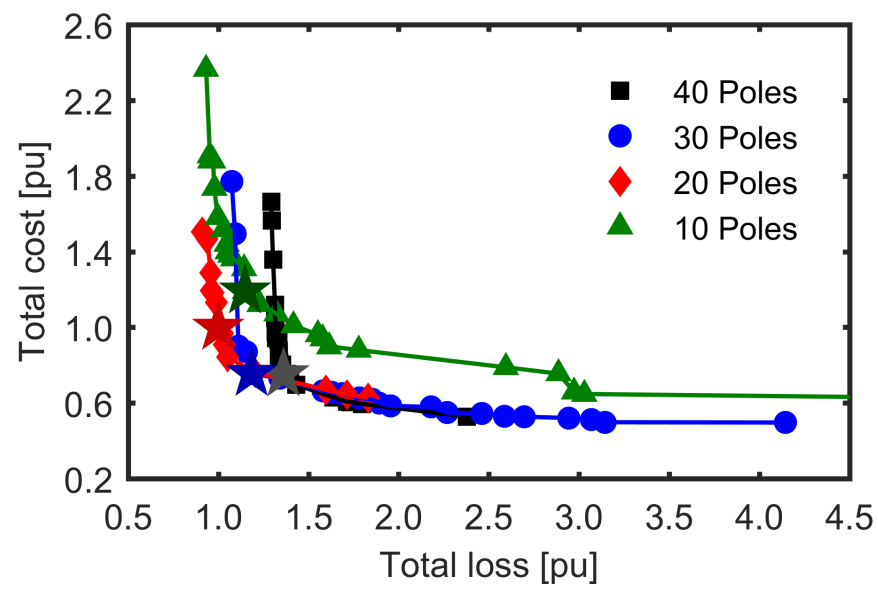

Fig. 9. Pareto fronts obtained for the machines with different pole count, employing 3D FEA models. A representative design of each topology is marked with a star symbol $(\star)$. The per-unit system is based on the representative design with the highest efficiency which is the 20 pole topology marked with a red star symbol $(\star)$.

\section{B. Identification of Trends}

The representative optimum design for each pole count is selected to be on the knee point of the Pareto front, marked with a star in Fig. 9. The breakdown of active material mass, cost, and loss for these designs is shown in Table III and Fig. 10. Table III also includes the global performance parameters, i.e., power density, torque density, and goodness defined as $\mathrm{Nm}$ per loss unit, in order to provide comparison indices with other designs.

It is observed that for the optimum design with a lower number of poles and slots, the copper and steel mass increase. The PM mass is affected by several variables including the split ratio, overhang, PM thickness, and PM arc ratio and it comprises a smaller portion of the total mass.

It is also seen that copper losses of the knee point design reduce initially upon increasing pole count, and then increase. This trend may in part be explained by considering that as 


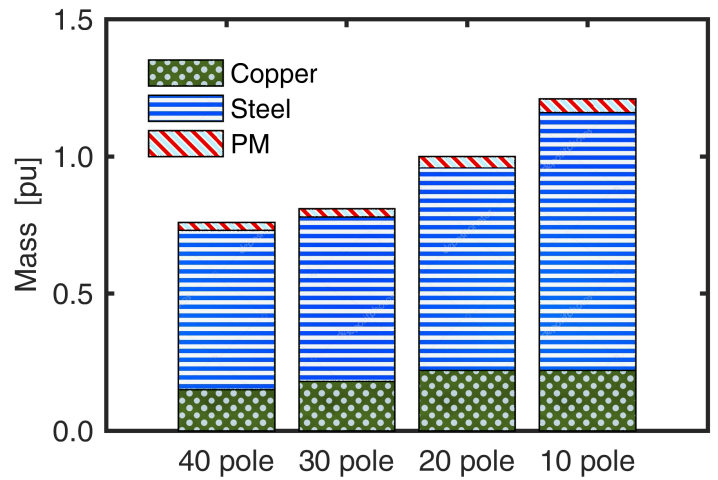

(a)

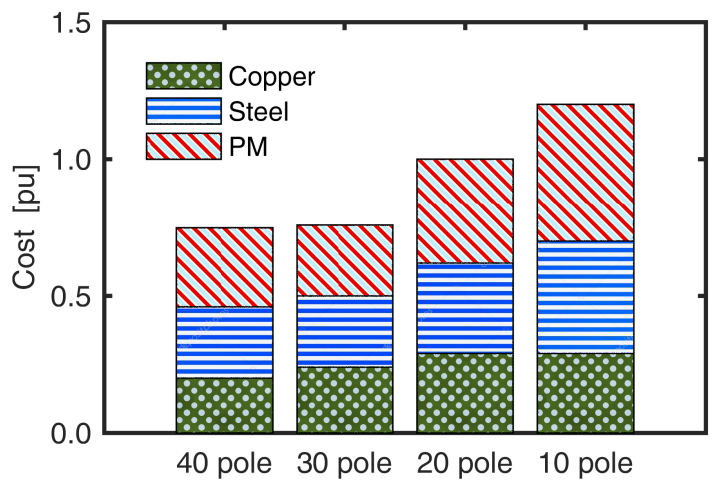

(b)

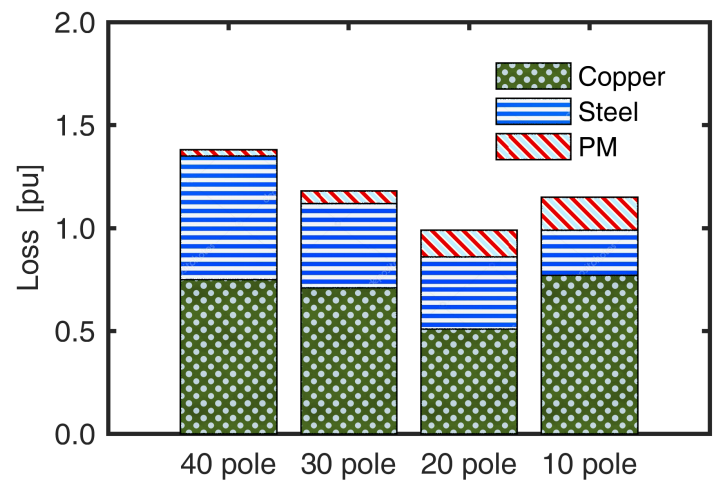

(c)

Fig. 10. The mass, cost, and loss components of the design on the knee point of the Pareto front obtained from the optimization studies with different number of poles.

the number of poles and slots reduces, the end turn length increases, while the current density reduces. The minimum copper loss is obtained for the design with 20 poles, given the specifications and ratings of this study. The core loss is lower for optimum designs with lower number of poles. This can be attributed to the reduced electrical frequency. The magnet loss increases for lower pole counts; magnet segmentation is not considered in the optimization process, therefore lower pole counts with larger pieces of magnets may increase the magnet eddy current losses. Magnet segmentation would affect the cost function which adds to the complications of setting up a systematic optimization algorithm. It also should be noted

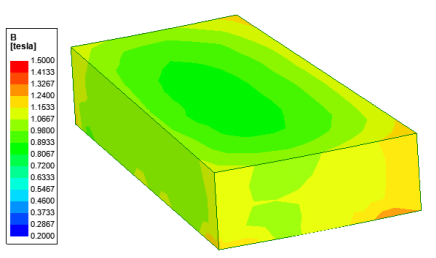

(a)

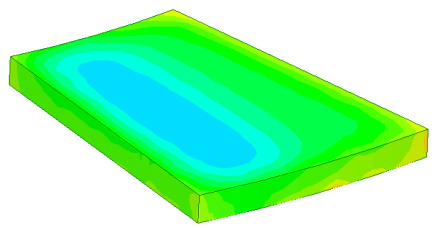

(c)

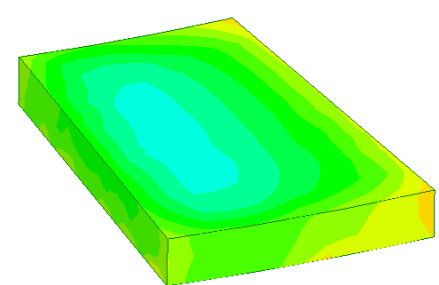

(b)

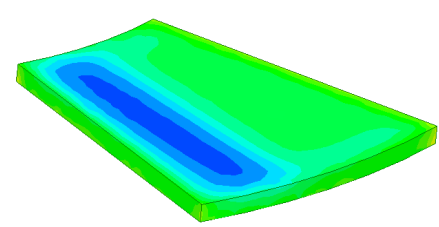

(d)
Fig. 11. Flux density distribution in magnets of the optimum designs with (a) 40, (b) 30, (c) 20, and (d) 10 poles at the rated load. Note that the color scale is identical and ranges from $0.2 \mathrm{~T}$ to $1.5 \mathrm{~T}$.

TABLE IV

MiNIMUM FLUX DENSITY WITHIN NEODYMIUM MAGNETS OF THE OPTIMUM DESIGNS SELECTED ON THE KNEE OF THE PARETO FRONT.

\begin{tabular}{lrrrr}
\hline Number of poles & 40 & 30 & 20 & 10 \\
\hline Open-circuit [T] & 0.57 & 0.45 & 0.39 & 0.30 \\
Load 1 pu [T] & 0.57 & 0.45 & 0.39 & 0.28 \\
Load 2 pu [T] & 0.57 & 0.41 & 0.36 & 0.20 \\
\hline
\end{tabular}

that, compared to the other two loss components, magnet loss is a smaller portion of the total losses.

In order to evaluate the PM demagnetization situation, the minimum flux density within the entire PM space is recorded and presented in Table IV. The evaluations are carried for open-circuit, rated and double the rated load. The flux density distribution at the rated operating condition is illustrated in Fig. 11. The optimization results suggest thicker magnets for the designs with higher pole count. Therefore, the lowest flux density of $0.3 \mathrm{~T}$ is observed in case of the machine with the lowest pole count and thinner magnets which have a lower permeance coefficient. The magnets of the machine with 40 poles have minimum flux density of $0.57 \mathrm{~T}$. The demagnetization curve of employed Neodymium PM exhibits a knee at about $0.35 \mathrm{~T}$ for $75^{\circ} \mathrm{C}$. The results show that for this design problem, optimum designs with higher pole count have a lower risk of demagnetization.

\section{Characteristics of Optimum Designs}

The box plot in Fig. 12 illustrates the distribution of variables for designs on the Pareto fronts. This plot can be used to identify certain characteristics of optimum designs as discussed in the following. The PM thickness of the optimal designs located on the Pareto front tends towards their smaller values and more so for lower pole counts. Generally, the PM arc ratios are larger for the lower pole counts. This implies that when the number of poles is lower, a design with thinner PMs and larger pole arc to pole pitch ratio will be more beneficial. The optimization algorithm reduces the thickness of 


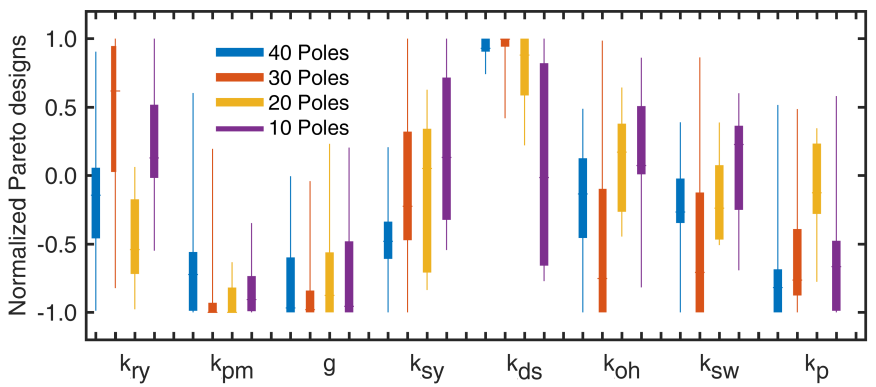

Fig. 12. Distribution of the design variables for the Pareto front designs obtained with different pole counts.

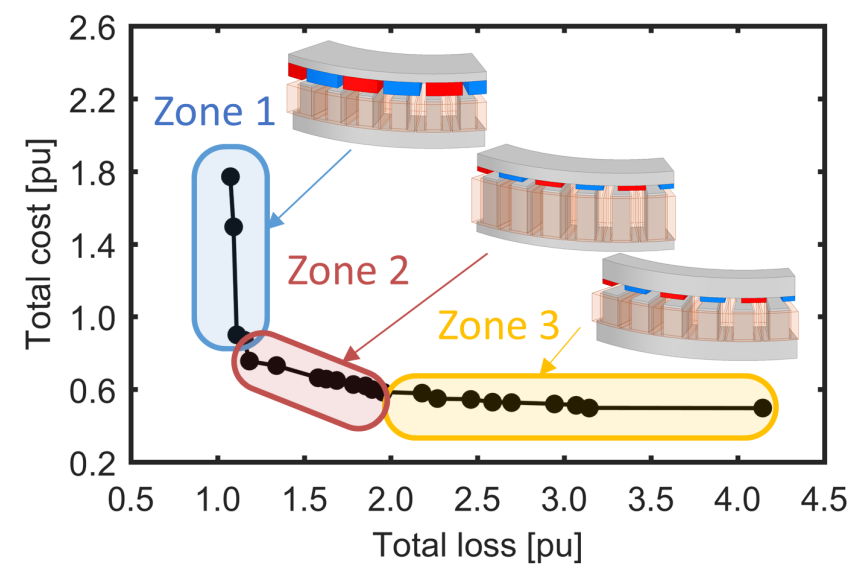

Fig. 13. Pareto front obtained for the machine with 30 poles, divided into three zones, the vertical section (zone 1) where further reduction in loss requires at least 5 times more increase in cost, the knee in the middle (zone 2), and the horizontal section (zone 3) where further reduction in cost requires at least 5 times more increase in loss.

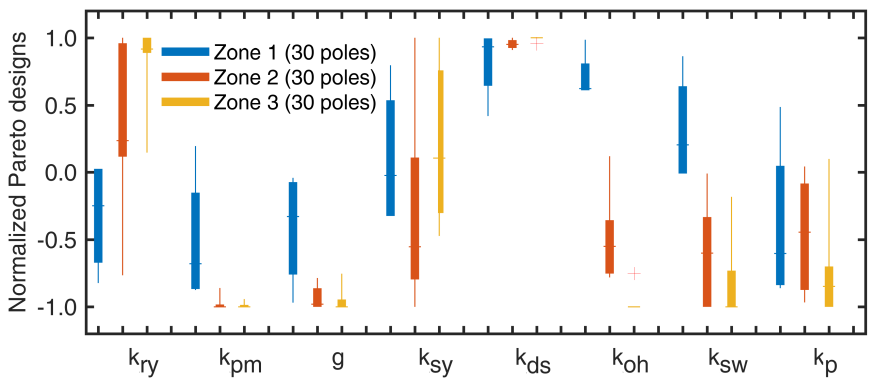

Fig. 14. Distribution of the design variables at different zones of the Pareto front for the topology with 30 poles. This topology is selected due to the its extended asymptotic Pareto front on both sides, illustrated in Fig. 13.

the PMs with wider arcs that may result in reduction of the PM volume and eddy losses. The stator yoke in optimum designs is generally larger for topologies with lower pole counts. This can be to reduce the flux density in the yoke and hence core loss.

The split ratio of the Pareto front designs is generally inclined toward their greater value and more so for higher pole and slot counts. A larger split ratio reduces the active material weight and cost and provides a larger tooth width in the inner diameter, mitigating the risk of saturation. The increased number of slots necessitates larger split ratio for core loss reduction.

To examine the extreme Pareto designs which are located on the tips of the Pareto front, the 30 pole topology is exemplified because of its extended and asymptotic Pareto front. The Pareto front is divided into three zones shown in Fig. 13.

The division of Pareto designs into three zones is conducted using the factor $T$, as explained by (5). Zone 1 is selected to be where further reduction of loss causes at least 5 times more increase in cost which corresponds to $T<0.2$. Zone 3 is where further reduction in cost leads to at least 5 times more increase in loss, corresponding to $T>5$. Zone 2 includes the Pareto designs in between. Therefore, designs in zone 1 and 3 are optimized with an emphasis on minimizing the loss and cost, respectively, while the designs in zone 2 display a preference of balance between the two objectives.

Based on Fig. 14, the high efficiency designs in zone 1 have a thinner yoke and a larger air-gap. However, the use of more magnet material results in costly designs. More efficient designs also have wider slots, smaller current density and copper loss. The less expensive, albeit less efficient, designs in zone 3 are achieved by reducing the PM thickness, the PM arc, and the overhang ratio, and also by increasing the split ratio.

Extreme cases of designs, with the lowest loss and with the lowest cost, along with the knee designs that achieves tradeoff between loss and cost are presented in Fig. 15. The design with the least loss in Fig. 15a has thick PMs and a thin yoke such that the cost is mostly associated with the PMs at about $78 \%$ of the total cost. Copper and core losses are both equal, at about $17 \mathrm{~W}$. The design in Fig. 15b has the minimum cost and employs very thin PMs and a very thick yoke. The copper loss accounts for most of the total loss with $134 \mathrm{~W}$ out of $140 \mathrm{~W}$, while the cost is distributed evenly among the materials. The results illustrate that the designs in the knee zone, i.e. the best trade-off region, have balanced cost and loss components, a characteristic typically associated with robust optimal designs.

\section{PROTOTYPING AND EXPERIMENTAL VALIDATION}

All the designs evaluated for the four slot/pole combinations along with the Pareto front obtained are represented in Fig. 16. A lower loss design can be obtained from the 20 pole configuration, while a less expensive design can be achieved with 30 or 40 poles. A machine with 20 poles and 24 slots which achieves the ultra high efficiency in excess of $94 \%$ is selected for prototyping and marked with a star in Fig. 16. The manufactured prototype is presented in Fig. 17.

The estimated performance using the 3D FEA model is verified with measurements and represented in Table V [24]. The spinning loss includes mechanical losses, such as friction and windage, stator core losses, and PM losses. The calculated efficiency uses copper and core losses obtained from the FEA and $6 \mathrm{~W}$ mechanical losses. The results for the measurements and the FEA are in agreement and serve as the basis for the satisfactory validation of the study.

The performance of the motor is investigated at different speed and torque values. The efficiency map is measured and 


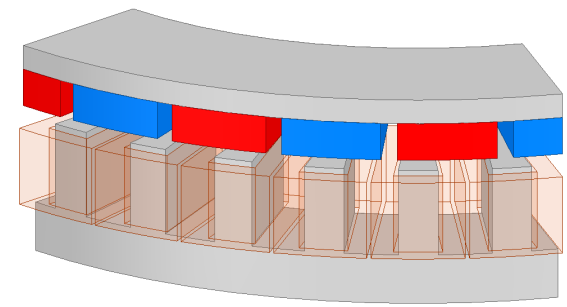

(a)

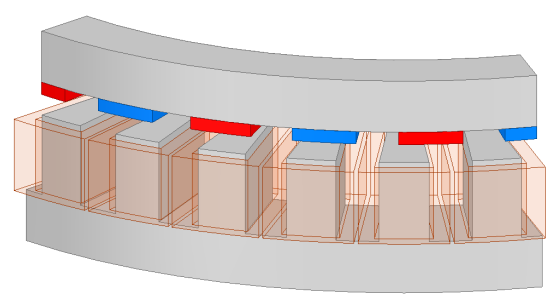

(b)

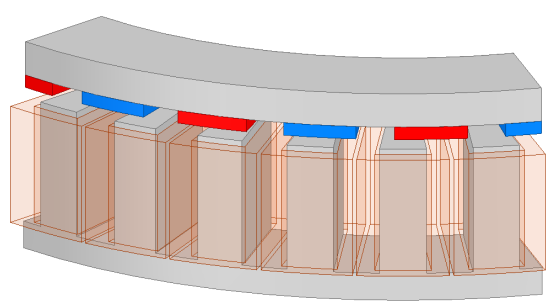

(c)

Fig. 15. The optimum designs obtained for the configuration with 30 poles. (a) The design with minimum loss on the Pareto front, (b) the design with minimum cost on the Pareto front, (c) the design on the knee of the Pareto front with a trade-off between cost and loss.

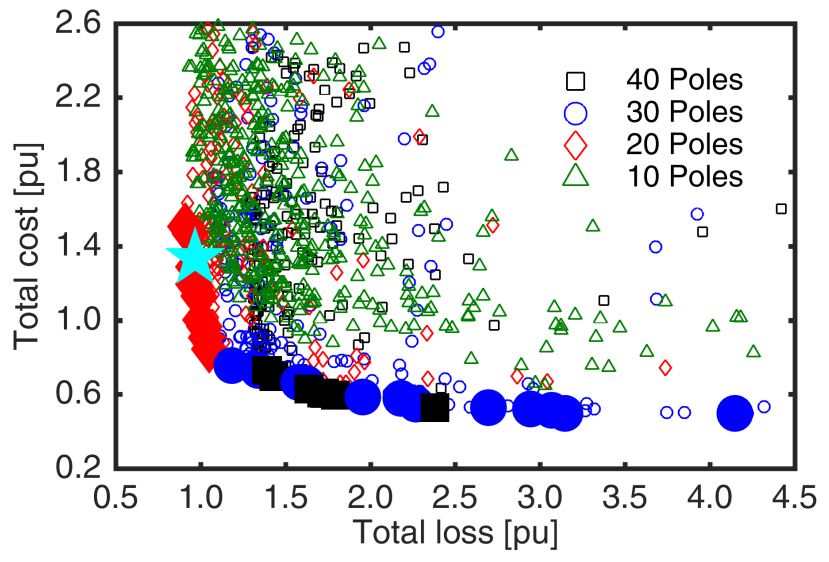

Fig. 16. The overall Pareto front (filled markers) and all of the evaluated designs (hollow markers). The prototyped design is selected to have a high efficiency, marked with a star symbol $(\star)$.

TABLE V

THE EXPERIMENTAL AND CALCULATED RESULTS FOR THE ULTRA HIGH EFFICIENCY AXIAL FLUX SPM MACHINE RATED AT 0.75 HP. THE CALCULATED VALUE OF THE EFFICIENCY USES 3D FEA RESULTS AND A 6 W MECHANICAL LOSS COMPONENT.

\begin{tabular}{llrr}
\hline & & Calculated & Measured \\
\hline Torque Constant & {$[\mathrm{Nm} / \mathrm{A}]$} & 1.4 & 1.4 \\
Phase resistance & {$[\Omega]$} & 0.39 & 0.37 \\
Conductor loss & {$[\mathrm{W}]$} & 17.4 & 15.3 \\
Core loss & {$[\mathrm{W}]$} & 10.9 & - \\
Mechanical loss & {$[\mathrm{W}]$} & 6 & - \\
Spinning loss & {$[\mathrm{W}]$} & - & 17.3 \\
Total loss & {$[\mathrm{W}]$} & 34.3 & 32.6 \\
Efficiency & {$[\%]$} & 94.5 & 94.3 \\
\hline
\end{tabular}

plotted in Fig. 18. The calculated efficiency map within the same speed and torque range matches satisfactorily with the measurements. The performance of the machine at the rated torque and speed is marked.

\section{CONCLUSION}

This paper systematically identifies the absolute performance limits that can be achieved by fractional horse power AFPM machines with surface magnets within a given envelope. This necessitates the study of a large number of designs spanning a wide design space, and having different slot-pole combinations, and the problem is further complicated due to the three-dimensional nature of the machine geometry. In

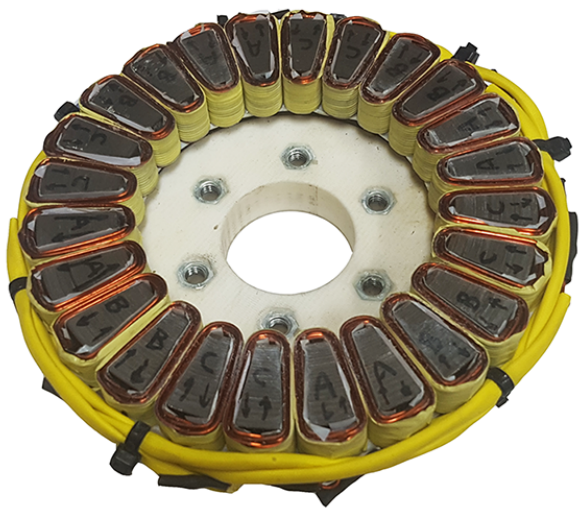

(a)

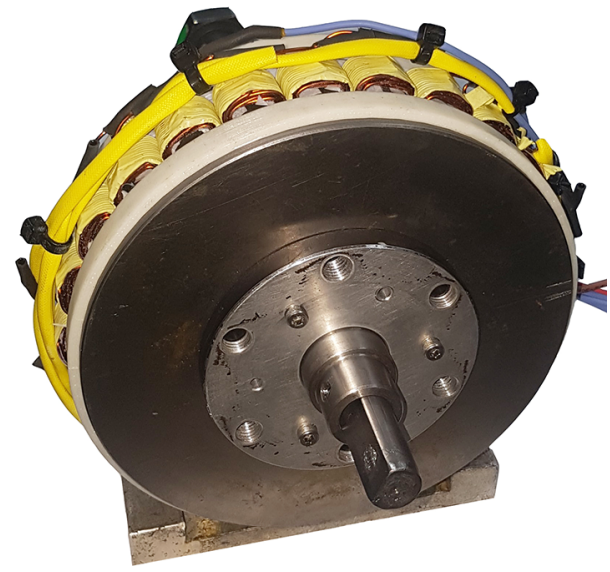

(b)

Fig. 17. The ultra high efficiency AFPM machine, rated for $5.4 \mathrm{Nm}$ at 1050 rpm, selected for prototyping based on the comprehensive optimization study with 3D FEA models. (a) The stator with a core OD of approx. $170 \mathrm{~mm}$ and (b) the assembled motor.

this regard, a new two-level surrogate assisted multi-objective optimization algorithm which enables this large scale study is utilized. The algorithm greatly improves the computational efficiency achieved through a significant reduction in the number of 3D finite element evaluations to less than two hundred, while a conventional optimization method would have required thousands of FEA design evaluations. This economizes the computational resources and time, thereby making the optimization of such expensive problems feasible. The paper also proposes a systematic method for selecting the optimum designs for multi-objective optimization problems. 


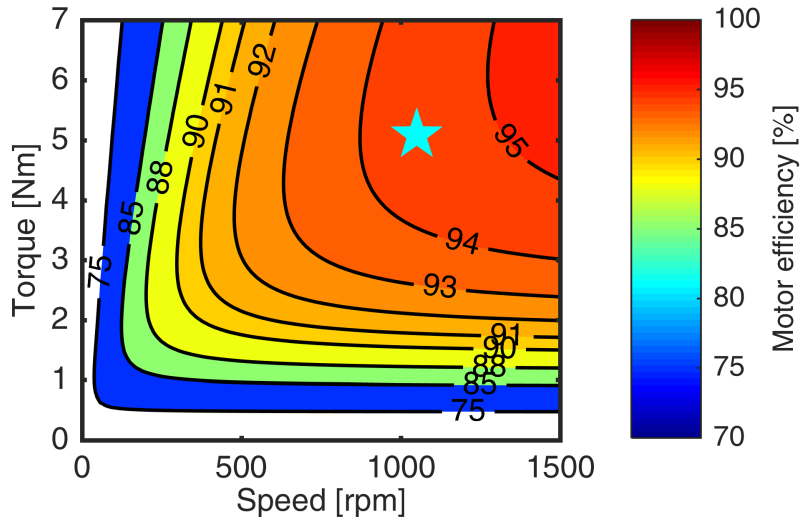

Fig. 18. The measured efficiency map of the prototyped machine. The performance of the machine at its rated torque and speed is marked with a star symbol $(\star)$.

Approximately horizontal and vertical lines obtained on the extremes of the Pareto front indicate that within the geometrical limitations further improvement in efficiency and cost is not probable. The results confirmed that the designs with the best trade-off between the two objectives of loss and mass split the core and copper losses nearly equally.

The combined Pareto front with pole counts ranging from 10 to 40 illustrates that the highest efficiency is obtained for designs employing 20 poles. An ultra high efficiency axial flux SPM design with 20 poles rated for $0.75 \mathrm{hp}$ is prototyped, demonstrating a measured efficiency of $94.3 \%$.

Furthermore, the effect of the pole count on the optimum design variables is examined to find a relative trend. For instance, it is observed that thinner PMs with a larger pole arc to pole pitch ratio are generally more beneficial for lower pole counts. Within the studied frame size and ratings, a bigger air-gap, a thinner yoke, and a larger slot width result in more efficient, albeit more expensive, designs. Learning such trends can serve as a basis for developing generalized design rules and as a reference for the preliminary stages of the optimum design process, ultimately making the optimization results more accurate as well as computationally affordable.

\section{ACKNOWLEDGMENT}

The support of Regal Beloit Corp., University of Kentucky, the L. Stanley Pigman endowment and the SPARK program, and ANSYS Inc. is gratefully acknowledged.

\section{REFERENCES}

[1] M. Rosu, P. Zhou, D. Lin, D. M. Ionel, M. Popescu, F. Blaabjerg, V. Rallabandi, and D. Staton, Multiphysics Simulation by Design for Electrical Machines, Power Electronics and Drives. Wiley-IEEE Press, 2017.

[2] S. Santoso and H. W. Beaty, Standard Handbook For Electrical Engineers, Seventeenth Edition. McGraw Hill, 2018, pp. 919-960.

[3] N. Bianchi, S. Bolognani, M. D. Pre, and G. Grezzani, "Design considerations for fractional-slot winding configurations of synchronous machines," IEEE Transactions on Industry Applications, vol. 42, no. 4, pp. 997-1006, July 2006.

[4] N. Taran, V. Rallabandi, G. Heins, and D. M. Ionel, "Coreless and conventional axial flux permanent magnet motors for solar cars," IEEE Transactions on Industry Applications, vol. 54, no. 6, pp. 5907-5917, Nov 2018.
[5] J. Cros and P. Viarouge, "Synthesis of high performance PM motors with concentrated windings," IEEE Transactions on Energy Conversion, vol. 17, no. 2, pp. 248-253, Jun 2002.

[6] D. Ishak, Z. Q. Zhu, and D. Howe, "Permanent-magnet brushless machines with unequal tooth widths and similar slot and pole numbers," IEEE Transactions on Industry Applications, vol. 41, no. 2, pp. 584-590, March 2005.

[7] K. F. Konecny, "Compact three-phase permanent magnet rotary machine having low vibration and high performance," U.S. Patent 4774 428A, May 15, 1987.

[8] T. Katsuma and M. Kitoh, "Brushless motor having permanent magnet rotor and salient pole stator," U.S. Patent 4719378A, 23, 1984.

[9] K. Wang, Z. Q. Zhu, and G. Ombach, "Synthesis of high performance fractional-slot permanent-magnet machines with coil-pitch of two slotpitches," IEEE Transactions on Energy Conversion, vol. 29, no. 3, pp. 758-770, Sept 2014.

[10] K. Ahsanullah, R. Dutta, and M. F. Rahman, "Analysis of low-speed IPMMs with distributed and fractional slot concentrated windings for wind energy applications," IEEE Transactions on Magnetics, vol. 53, no. 11 , pp. $1-10$, Nov 2017.

[11] C. Tang, W. L. Soong, G. S. Liew, and N. Ertugrul, "Effect of pole and slot number changes on the performance of a surface PM machine," in 2012 XXth International Conference on Electrical Machines, Sept 2012, pp. 220-227.

[12] Y. S. Chen, Z. Q. Zhu, and D. Howe, "Vibration of PM brushless machines having a fractional number of slots per pole," IEEE Transactions on Magnetics, vol. 42, no. 10, pp. 3395-3397, Oct 2006.

[13] C. C. Mi, G. R. Slemon, and R. Bonert, "Minimization of iron losses of permanent magnet synchronous machines," IEEE Transactions on Energy Conversion, vol. 20, no. 1, pp. 121-127, March 2005.

[14] S. M. Castano, J. W. Jiang, B. Bilgin, A. Sathyan, H. Dadkhah, and A. Emadi, "An investigation of slot-pole combinations for interior permanent magnet synchronous machines with different magnet topologies," in 2017 IEEE International Electric Machines and Drives Conference (IEMDC), May 2017, pp. 1-8.

[15] Y. Liu, Z. Zhu, C. Gan, S. Brockway, and C. Hilton, "Comparison of optimal slot/pole number combinations in fractional slot permanent magnet synchronous machines having similar slot and pole numbers," in International Conference on Power Electronics, Machines and Drives (PEMD), Apr. 2018.

[16] N. Taran, V. Rallabandi, G. Heins, and D. M. Ionel, "Exploring the efficiency and cost limits of fractional hp axial flux PM machine designs," in 2018 IEEE Energy Conversion Congress and Exposition (ECCE), Sep. 2018, pp. 3272-3277.

[17] Y. Duan and D. M. Ionel, "Nonlinear scaling rules for brushless PM synchronous machines based on optimal design studies for a wide range of power ratings," IEEE Transactions on Industry Applications, vol. 50, no. 2, pp. 1044-1052, March 2014.

[18] A. Fatemi, D. M. Ionel, N. A. O. Demerdash, and T. W. Nehl, "Optimal design of IPM motors with different cooling systems and winding configurations," IEEE Transactions on Industry Applications, vol. 52, no. 4, pp. 3041-3049, July 2016.

[19] N. Taran, D. M. Ionel, and D. G. Dorrell, "Two-level surrogate-assisted differential evolution multi-objective optimization of electric machines using 3-d fea," IEEE Transactions on Magnetics, vol. 54, no. 11, pp. 1-5, Nov 2018.

[20] C. E. Rasmussen and C. K. I. Williams, Gaussian Processes for Machine Learning. MIT Press, 2006.

[21] T. W. Simpson, T. M. Mauery, J. J. Korte, and F. Mistree, "Kriging models for global approximation in simulation-based multidisciplinary design optimization," AIAA Journal, vol. 39, no. 12, pp. 2233-2241, 2001.

[22] C. A. C. Coello, G. B. Lamont, D. A. Van Veldhuizen et al., Evolutionary algorithms for solving multi-objective problems. Springer, 2007, vol. 5.

[23] Y. Duan and D. M. Ionel, "A review of recent developments in electrical machine design optimization methods with a permanent magnet synchronous motor benchmark study," in 2011 IEEE Energy Conversion Congress and Exposition, November 2011.

[24] G. Heins, D. M. Ionel, D. Patterson, S. Stretz, and M. Thiele, "Combined experimental and numerical method for loss separation in permanentmagnet brushless machines," IEEE Transactions on Industry Applications, vol. 52, no. 2, pp. 1405-1412, March 2016. 